\title{
New Classes of the Moderate Deviation Functions
}

\author{
${ }^{*}$ Jana Špirková ${ }^{b}$ Humberto Bustince $^{b}$, Javier Fernandez $^{b}$ and Mikel Sesma-Sara ${ }^{b}$ \\ ${ }^{a}$ Department of Quantitative Methods and Information Systems, Faculty of Economics, \\ Matej Bel University in Banská Bystrica, Tajovského 10, 97590 Banská Bystrica, \\ Slovakia, jana.spirkova@umb.sk \\ ${ }^{b}$ Department of Statistics, Computer Science and Mathematics, Institute of Smart Cities, \\ Public University of Navarra, Campus Arrosadia, s/n, 31.006 Pamplona, Spain, \\ bustince@unavarra.es, fcojavier.fernandez@unavarra.es, mikel.sesma@unavarra.es
}

\begin{abstract}
At present, in the field of aggregation of various input values, attention is focused on the construction of aggregation functions using other functions that can affect the resulting aggregated value. This resulting value should characterize the properties of the individual input values as accurately as possible. Attention is also paid to aggregation using the so-called moderate deviation function. Using this function in aggregation ensures that all properties of aggregation functions are preserved. This work offers constructions of the moderate deviation functions using negations and automorphisms on the symmetric interval $[-1,1]$ and a general closed interval $[a, b] \subset[-\infty, \infty]$.
\end{abstract}

Keywords: Moderate deviation function, Automorphism, Negation, Aggregation function, Transformation.

\section{Introduction}

Almost 50 years ago, Daróczy introduced the construction of specific means using the so-called deviation function, [4]. These functions measure the deviation between two real values and aggregate a number of input values into one single output. The main goal of this aggregation is to obtain such an output value that characterizes the whole set of inputs as concisely as possible. However, mentioned Daróczy's means do not always maintain monotonicity, i.e., they are not, in general, aggregation functions, $[5,6,10]$. Therefore, the authors in [5, 6] introduced a so-called moderate deviation function, which ensures that the aggregation functions based on the moderate deviation functions meet all the properties of aggregation functions. At present, researches offer various constructions of aggregation functions, which are based on the use of the mentioned moderate deviation functions, $[1,5,6,11]$.

Our intention is also to offer constructions of aggregation functions through a moderate deviation function on the interval $[-1,1]$ and on a general interval $[a, b]$, which is constructed utilizing negations and automorphisms. For this construction we decided to use a specific negation $N(x)=-x$ in the interval $[-1,1]$ and then a linear isomorphism from the interval $[a, b]$ to $[-1,1]$, i.e., $T(x)=\frac{2}{b-a} x-\frac{a+b}{b-a}$. To such a construction of the moderate deviation function led us discussions of papers [3] and [9]. According to the authors of the mentioned papers, it is known that people also manage other types of scales, including bipolar, which can be expressed in the interval $[-1,1]$. This scale is particularly interesting because it supports the representation of the symmetrical phenomena we encounter in human behaviour. Thus, the symmetry of the interval $[-1,1]$ would better correspond to the numerical representation of the uncertainty than the asymmetry of the unit interval $[0,1]$. This interval is also taken into account because it has an algebraic structure as a circle in [7] and [8].

The paper is organized as follows. In Section 2, we give basic definitions concerning negation and moderate deviation functions. In Section 3, we introduce statements which give us possibilities of construction of moderate deviation functions on the interval $[-1,1]$. In Section 4, we add our statements of construction of moderate deviation functions on a general closed interval $[a, b]$. Our statements are illustrated by examples. Finally, some remarks are given in conclusions.

\section{Preliminaries}

In the paper the following notations and definitions are used.

We denote by $\mathbf{I}=[a, b] \subset \mathbf{R}=[-\infty, \infty]$ a closed interval. In this way, $\mathbf{I}^{n}=\left\{\mathbf{x}=\left(x_{1}, \ldots, x_{n}\right) \mid x_{i} \in \mathbf{I}, i=\right.$ 
$1, \ldots, n\}$ is the set of all vectors $\mathbf{x}$ whose components lie in the interval $\mathbf{I}$. Considering $\mathbf{x}, \mathbf{y} \in \mathbf{I}^{n}, \mathbf{x}=$ $\left(x_{1}, \ldots, x_{n}\right), \mathbf{y}=\left(y_{1}, \ldots, y_{n}\right)$, we say that $\mathbf{x} \leq \mathbf{y}$ if and only if $x_{i} \leq y_{i}$ for each $i=1, \ldots, n$.

Throughout the paper, we consider automorphisms and negation on the interval $[-1,1]$, because even in the construction of the deviation function on the interval $[a, b]$, we use the transformation using linear isomorphism to $[-1,1]$.

Definition 1. An automorphism $\phi$ is a bijective and increasing function $\phi:[-1,1] \rightarrow[-1,1]$.

Definition 2. A negation $N$ is a decreasing function $N:[-1,1] \rightarrow[-1,1]$ such that $N(-1)=1$ and $N(1)=$ -1 .

Definition 3. (see, e.g., [2]) (Strict negation) A function $N$ defined on $[-1,1]$ is called a strict negation, if its range is also $[-1,1]$ and it is strictly monotone decreasing.

Definition 4. (see, e.g., [2]) (Strong negation) A function $N$ defined on $[-1,1]$ is called a strong negation, if it is strictly decreasing and involutive (i.e., $N(N(x))=$ $x$ for all $x \in[-1,1])$.

Theorem 1. (see, e.g., [2]) A function $N:[-1,1] \rightarrow$ $[-1,1]$ is a strong negation if and only if there exists an automorphism $\phi:[-1,1] \rightarrow[-1,1]$ such that $N(x)=$ $\phi^{-1}(-\phi(x))$.

We would like to emphasize that in our paper we offer only moderate deviation function constructions using negation in the form $N(x)=-x$.

Now, we give two basic definitions of moderate deviation function and so-called global moderate deviation function.

Definition 5. ([1], [11]) A function $D: \mathbf{I}^{2} \rightarrow \mathbf{R}$ is called a moderate deviation function, if and only if it satisfies the following conditions:

(i) for every $x \in \mathbf{I}, D(x, \cdot): \mathbf{I} \rightarrow \mathbf{R}$ is increasing (not necessarily strictly);

(ii) for every $y \in \mathbf{I}, D(\cdot, y): \mathbf{I} \rightarrow \mathbf{R}$ is decreasing (not necessarily strictly);

(iii) $D(x, y)=0$ if and only if $x=y, x \in \mathbf{I}, y \in \mathbf{I}$.

Definition 6. [11] Consider a moderate deviation function $D: \mathbf{I}^{2} \rightarrow \mathbf{R}$ and $n \in \mathbf{N}$. Then the function $\mathscr{G}: \mathbf{I}^{n} \times \mathbf{I} \rightarrow \mathbf{R}$ given by

$$
\mathscr{G}(\mathbf{x}, y)=\sum_{i=1}^{n} D\left(x_{i}, y\right)
$$

is called a global moderate deviation function.
However, we would like to point out that equation

$$
\mathscr{G}(\mathbf{x}, y)=\sum_{i=1}^{n} D\left(x_{i}, y\right)=0
$$

need not have a solution, or it may have more solutions, depending on particular moderate deviation function. For more information, see [11].

We would like to emphasize that in our paper we introduce such constructions of the moderate deviation functions which give us exactly one solution.

\section{Moderate deviation functions based on negation and automorphism}

In this section, we provide a few propositions and theorems corresponding to the creation of various types of deviation functions based on automorphisms and negations. Particularly we focus on generating the moderate deviation functions $D:[-1,1]^{2} \rightarrow[-1,1]$ using automorphisms and negation $N(x)=-x$ on $[-1,1]$.

Proposition 1. Let $\phi_{1}, \phi_{2}:[-1,1] \rightarrow[-1,1]$ be automorphisms, $\phi_{1}(0)=0$, and $N:[-1,1] \rightarrow[-1,1]$, $N(x)=-x$. Then a function $D:[-1,1]^{2} \rightarrow[-1,1]$ given by

$$
D(x, y)=\phi_{1}^{-1}\left(\max \left\{-1, \min \left\{1, \phi_{2}(N(x))+N\left(\phi_{2}(N(y))\right)\right\}\right\}\right)
$$

is a moderate deviation function.

Proof. The result directly follows from properties of the automorphism and negation.

Proposition 2. Let $\phi_{1}, \phi_{2}:[-1,1] \rightarrow[-1,1]$ be automorphisms, $\phi_{1}(0)=0$, and $N:[-1,1] \rightarrow[-1,1]$, $N(x)=-x$. Then a function $D:[-1,1]^{2} \rightarrow[-1,1]$ given by

$$
D(x, y)=\phi_{1}^{-1}\left(\max \left\{-1, \min \left\{1, N\left(\phi_{2}(x)\right)+\phi_{2}(y)\right\}\right\}\right)
$$

is a moderate deviation function.

Proof. The proof directly follows from properties of the automorphisms and from the negation used.

Theorem 2. Let $\phi:[-1,1] \rightarrow[-1,1]$ be an automorphism, $N:[-1,1] \rightarrow[-1,1], N(x)=-x$, and $F:$ $[-1,1]^{2} \rightarrow[-1,1]$ increasing function in both components and it satisfies condition $F(-x, y)=0$ if and only if $x=y$. Then a function $D:[-1,1]^{2} \rightarrow[-1,1]$ given by

$$
D(x, y)=\phi^{-1}(F(N(\phi(x)), \phi(y)))
$$

is a moderate deviation function. 
Proof. Consider function (5). If $\phi$ is increasing then inverse function $\phi^{-1}$ is also increasing. We can see immediately that function $F(N(\phi(x)), \phi(y))$ is increasing in the second component and decreasing in the first one.

Now, it is enough to show that $D(x, y)=0$ if and only if $x=y$. It follows directly from the special property of the function $F$.

Corollary 1. Let $\phi:[-1,1] \rightarrow[-1,1]$ be an automorphism, $N:[-1,1] \rightarrow[-1,1], N(x)=-x$, and $F$ : $[-1,1]^{2} \rightarrow[-1,1]$ increasing function in both components and it satisfies condition $F(-x, y)=0$ if and only if $x=y$. Moreover, let $c \in] 0,1]$ be an arbitrary constant. Then a function $D:[-1,1]^{2} \rightarrow[-1,1]$ given by

$$
D(x, y)=\phi^{-1}(F(c \cdot N(\phi(x)), c \cdot \phi(y)))
$$

is a moderate deviation function.

Corollary 2. Let $\phi:[-1,1] \rightarrow[-1,1]$ be an automorphism, $N:[-1,1] \rightarrow[-1,1], N(x)=-x$, and $F:$ $[-1,1]^{2} \rightarrow[-1,1]$ decreasing function in both components and it satisfies condition $F(-x, y)=0$ if and only if $x=y$. Moreover, let $d \in[-1,0[$ be an arbitrary constant. Then a function $D:[-1,1]^{2} \rightarrow[-1,1]$ given by

$$
D(x, y)=\phi^{-1}(F(d \cdot N(\phi(x)), d \cdot \phi(y)))
$$

is a moderate deviation function.

Theorem 3. Let $\phi:[-1,1] \rightarrow[-1,1]$ be an automorphism, $N:[-1,1] \rightarrow[-1,1], N(x)=-x$, and $F:$ $[-1,1]^{2} \rightarrow[-1,1]$ decreasing function in both components and it satisfies condition $F(x,-y)=0$ if and only if $x=y$. Then a function $D:[-1,1]^{2} \rightarrow[-1,1]$ given by

$$
D(x, y)=\phi^{-1}(F(\phi(x), N(\phi(y))))
$$

is a moderate deviation function.

Proof. Similarly to proof of Theorem 2.

Corollary 3. Let $\phi:[-1,1] \rightarrow[-1,1]$ be an automorphism, $N:[-1,1] \rightarrow[-1,1], N(x)=-x$, and $F:$ $[-1,1]^{2} \rightarrow[-1,1]$ decreasing function in both components and it satisfies condition $F(x,-y)=0$ if and only if $x=y$. Moreover, let $k \in] 0,1]$ be an arbitrary constant. Then a function $D:[-1,1]^{2} \rightarrow[-1,1]$ given by

$$
D(x, y)=\phi^{-1}(F(k \cdot \phi(x), k \cdot N(\phi(y))))
$$

Corollary 4. Let $\phi:[-1,1] \rightarrow[-1,1]$ be an automorphism, $N:[-1,1] \rightarrow[-1,1], N(x)=-x$, and $F$ : $[-1,1]^{2} \rightarrow[-1,1]$ increasing function in both components and it satisfies condition $F(x,-y)=0$ if and only if $x=y$. Moreover, let $l \in[-1,0[$ be an arbitrary constant. Then a function $D:[-1,1]^{2} \rightarrow[-1,1]$ given by

$$
D(x, y)=\phi^{-1}(F(l \cdot \phi(x), l \cdot N(\phi(y))))
$$

is a moderate deviation function.

Example 1. Consider $\phi_{1}, \phi_{2}:[-1,1] \rightarrow[-1,1]$ automorphisms in the form $\phi_{1}(x)=x^{2 k+1} ; k \in Z_{0}^{+}$; and $\phi_{2}(x)=x^{2 t+1} ; t \in Z_{0}^{+}$. These automorphisms may or may not be the same. Moreover, consider $N$ the negation given by $N(x)=-x$. Then by Proposition 1

$$
D(x, y)=\phi_{1}^{-1}\left(\max \left\{-1, \min \left\{1, \phi_{2}(-x)-\phi_{2}(-y)\right\}\right\}\right)
$$

is a moderate deviation function. Indeed, function (11) meets the conditions for moderate deviation function.

Let us examine function (11)

$$
D(x, y)=\left(\max \left\{-1, \min \left\{1, y^{2 t+1}-x^{2 t+1}\right\}\right\}\right)^{\frac{1}{2 k+1}} .
$$

To show properties of the moderate deviation function defined by Definition 5, it is sufficient to find out the values of partial derivatives according to components $x$ and $y$. The fulfilment of the third condition can be seen directly from the function. Hence, $D(x, y)$ is a moderate deviation function.

In Figure 1, we show graphical representation of the moderate deviation function given by (12) for $t=1$ and $k=0$.

Using global moderate deviation function (1) and equality (2), for $k=0$ and $t=1$, we obtain the solution in the form

$$
y^{*}=\sqrt[3]{\frac{\sum_{i=1}^{n} x_{i}^{3}}{n}} .
$$

is a moderate deviation function. 


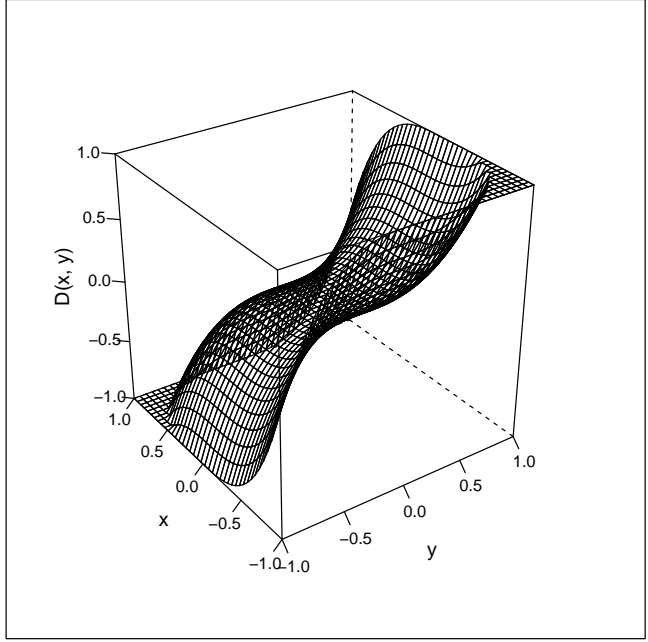

Figure 1: Moderate deviation function (12), $t=1$, $k=0$

Example 2. Consider automorphism $\phi:[-1,1] \rightarrow$ $[-1,1]$ in the form $\phi(x)=x^{3}, N(x)=-x$ and function $F:[-1,1]^{2} \rightarrow[-1,1] ; F(x, y)=\frac{x+y}{2}$. Then, by Theorem 2, we get a moderate deviation function as follows

$$
D(x, y)=\sqrt[3]{\frac{y^{3}-x^{3}}{2}} .
$$

All the conditions that are needed for this function to be a moderate deviation function are satisfied. For $n=2$ using equation (2) we get solution

$$
y^{*}=\sqrt[3]{\frac{x_{1}^{3}+x_{2}^{3}}{2}} .
$$

If $\phi(x)=i d$ and $\mathbf{x}=\left(x_{1}, \ldots, x_{n}\right)$ then $y^{*}$ is the arithmetic mean.

\section{Moderate deviation function on a general closed interval}

All the previous propositions and theorems can be applied to a general closed interval $\mathbf{I}=[a, b] \subset \mathbf{R}=$ $[-\infty, \infty]$. The deviation function for input values from the interval $[a, b]$ can be constructed using the following statements by applying a linear isomorphism between intervals $[a, b]$ and $[-1,1]$ in the form $T(x)=$ $\frac{2}{b-a} x-\frac{a+b}{b-a}$.
Proposition 3. Let $\phi_{1}, \phi_{2}:[-1,1] \rightarrow[-1,1]$ be automorphisms, $\phi_{1}(0)=0, N:[-1,1] \rightarrow[-1,1], N(x)=$ $-x$, be a strong negation and $T(x)=\frac{2}{b-a} x-\frac{a+b}{b-a}$ be $a$ linear isomorphism from interval $[a, b]$ to $[-1,1]$. Then a function $D:[a, b]^{2} \rightarrow[-1,1]$ given by

$D(x, y)=\phi_{1}^{-1}\left(\max \left\{-1, \min \left\{1, \phi_{2}(N(T(x)))+N\left(\phi_{2}(N(T(y)))\right)\right\}\right\}\right)$

is a moderate deviation function.

Proof. The result directly follows from properties of the automorphism and negation.

Proposition 4. Let $\phi_{1}, \phi_{2}:[-1,1] \rightarrow[-1,1]$ be automorphisms, $\phi_{1}(0)=0, N:[-1,1] \rightarrow[-1,1], N(x)=$ $-x$, be a strong negation and $T(x)=\frac{2}{b-a} x-\frac{a+b}{b-a}$ be $a$ linear isomorphism from interval $[a, b]$ to $[-1,1]$. Then a function $D:[a, b]^{2} \rightarrow[-1,1]$ given by

$$
D(x, y)=\phi_{1}^{-1}\left(\max \left\{-1, \min \left\{1, N\left(\phi_{2}(T(x))\right)+\phi_{2}(T(y))\right\}\right\}\right)
$$

is a moderate deviation function.

Proof. The proof directly follows from properties of the automorphisms and from the negation used.

Theorem 4. Let $\phi:[-1,1] \rightarrow[-1,1]$ be an automorphism, $N:[-1,1] \rightarrow[-1,1], N(x)=-x$, and $F$ : $[-1,1]^{2} \rightarrow[-1,1]$ increasing function in both components and it satisfies condition $F(-x, y)=0$ if and only if $x=y$. Moreover, let $T(x)=\frac{2}{b-a} x-\frac{a+b}{b-a}$ be a linear isomorphism from interval $[a, b]$ to $[-1,1]$. Then $a$ function $D:[a, b]^{2} \rightarrow[-1,1]$ given by

$$
D(x, y)=\phi^{-1}(F(N(\phi(T(x))), \phi(T(y))))
$$

is a moderate deviation function.

Corollary 5. Let $\phi:[-1,1] \rightarrow[-1,1]$ be an automorphism, $N:[-1,1] \rightarrow[-1,1], N(x)=-x$, and $F:$ $[-1,1]^{2} \rightarrow[-1,1]$ increasing function in both components and it satisfies condition $F(-x, y)=0$ if and only if $x=y$. Moreover, let $T(x)=\frac{2}{b-a} x-\frac{a+b}{b-a}$ be $a$ linear isomorphism from interval $[a, b]$ to $[-1,1]$ and $c \in] 0,1]$ be an arbitrary constant. Then a function $D:[a, b]^{2} \rightarrow[-1,1]$ given by

$$
D(x, y)=\phi^{-1}(F(c \cdot N(\phi(T(x))), c \cdot \phi(T(y))))
$$

is a moderate deviation function.

Corollary 6. Let $\phi:[-1,1] \rightarrow[-1,1]$ be an automorphism, $N:[-1,1] \rightarrow[-1,1], N(x)=-x$, and $F$ : $[-1,1]^{2} \rightarrow[-1,1]$ decreasing function in both components and it satisfies condition $F(-x, y)=0$ if and 
only if $x=y$. Moreover, let $T(x)=\frac{2}{b-a} x-\frac{a+b}{b-a}$ be $a$ linear isomorphism from interval $[a, b]$ to $[-1,1]$ and $d \in[-1,0[$ be an arbitrary constant. Then a function $D:[a, b]^{2} \rightarrow[-1,1]$ given by

$$
D(x, y)=\phi^{-1}(F(d \cdot N(\phi(T(x))), d \cdot \phi(T(y))))
$$

is a moderate deviation function.

Theorem 5. Let $\phi:[-1,1] \rightarrow[-1,1]$ be an automorphism, $N:[-1,1] \rightarrow[-1,1], N(x)=-x$, and $F$ : $[-1,1]^{2} \rightarrow[-1,1]$ decreasing function in both components and it satisfies condition $F(x,-y)=0$ if and only if $x=y$. Moreover, let $T(x)=\frac{2}{b-a} x-\frac{a+b}{b-a}$ be a linear isomorphism from interval $[a, b]$ to $[-1,1]$. Then $a$ function $D:[a, b]^{2} \rightarrow[-1,1]$ given by

$$
D(x, y)=\phi^{-1}(F(\phi(T(x)), N(\phi(T(y)))))
$$

is a moderate deviation function.

Corollary 7. Let $\phi:[-1,1] \rightarrow[-1,1]$ be an automorphism, $N:[-1,1] \rightarrow[-1,1], N(x)=-x$, and $F:$ $[-1,1]^{2} \rightarrow[-1,1]$ decreasing function in both components and it satisfies condition $F(x,-y)=0$ if and only if $x=y$. Moreover, let $T(x)=\frac{2}{b-a} x-\frac{a+b}{b-a}$ be $a$ linear isomorphism from interval $[a, b]$ to $[-1,1]$ and $k \in] 0,1]$ be an arbitrary constant. Then a function $D:[a, b]^{2} \rightarrow[-1,1]$ given by

$$
D(x, y)=\phi^{-1}(F(k \cdot \phi(T(x)), k \cdot N(\phi(T(y)))))
$$

is a moderate deviation function.

Corollary 8. Let $\phi:[-1,1] \rightarrow[-1,1]$ be an automorphism, $N:[-1,1] \rightarrow[-1,1], N(x)=-x$, and $F$ : $[-1,1]^{2} \rightarrow[-1,1]$ increasing function in both components and it satisfies condition $F(x,-y)=0$ if and only if $x=y$. Moreover, let $T(x)=\frac{2}{b-a} x-\frac{a+b}{b-a}$ be $a$ linear isomorphism from interval $[a, b]$ to $[-1,1]$ and $l \in[-1,0[$ be an arbitrary constant. Then a function $D:[a, b]^{2} \rightarrow[-1,1]$ given by

$$
D(x, y)=\phi^{-1}(F(l \cdot \phi(T(x)), l \cdot N(\phi(T(y)))))
$$

is a moderate deviation function.

The following example provides the possibility of aggregation of the input values from the general interval $[a, b]$ by the linear isomorphism applied to the interval $[-1,1]$.

Example 3. Let us consider conditions and statements which are written in Theorem 4. Assume the automorphism $\phi:[-1,1] \rightarrow[-1,1], \phi(x)=x^{3}, N:[-1,1] \rightarrow$ $[-1,1], N(x)=-x$ and $F:[-1,1]^{2} \rightarrow[-1,1]$ in the form $F(x)=\frac{x+y}{2}$. Then the moderate deviation function $D:[3,7]^{2} \rightarrow[-1,1]$ is as follows (see Figure 2)

$$
D(x, y)=\sqrt[3]{\frac{\left(\frac{1}{2} y-\frac{5}{2}\right)^{3}-\left(\frac{1}{2} x-\frac{5}{2}\right)^{3}}{2}} .
$$

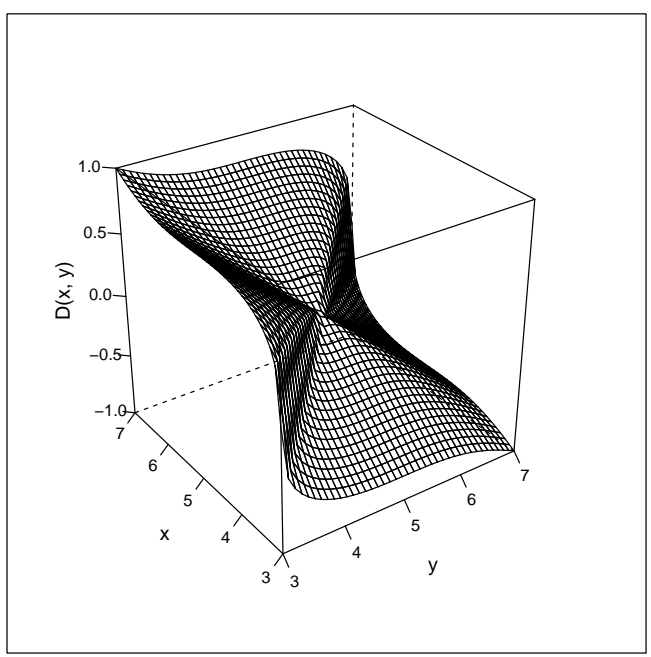

Figure 2: Moderate deviation function (23)

We can generate global moderate deviation function (1) using the moderate deviation function as follows

$$
\mathscr{G}(\mathbf{x}, y)=\sum_{i=1}^{n} \sqrt[3]{\frac{\left(\frac{1}{2} y-\frac{5}{2}\right)^{3}-\left(\frac{1}{2} x_{i}-\frac{5}{2}\right)^{3}}{2}}
$$

For example, for inputs $\mathbf{x}=(3.2,4.0,4.5,6.0,6.8)$, we give, using equality (2), as a unique solution $y^{*}=$ 4.50169 .

\section{Conclusions}

We have introduced new possibilities of construction of the moderate deviation functions using automorphisms and concrete negation in the form $N(x)=-x$ on the interval $[-1,1]$. Moreover, we have introduced possible constructions of the moderate deviation functions on the general interval $[a, b]$ using a linear isomorphism from an interval $[a, b]$ to $[-1,1]$. Our next goal is to generate the moderate deviation functions not only by means negation $N(x)=-x$, but also with general negation functions defined on the mentioned intervals.

\section{Acknowledgement}

We would like to take this opportunity to thank professor Radko Mesiar for his willingness to consult our solutions. 
The work of Jana Špirková has been supported by the Slovak Scientific Grant Agency VEGA no. 1/0150/21.

The work of Humberto Bustince, Javier Fernandez and Mikel Sesma-Sara has been supported by grand PID2019 - 108392GB-100 (AEI/10.13039/501100011033).

\section{References}

[1] A. H. Altalhi, J. I. Forcén, M. Pagola, E. Barrenechea, H. Bustince, Z. Takáč, Moderate deviation and restricted equivalence functions for measuring similarity between data, Information Sciences 501 (2019) pp. 19-29.

[2] G. Beliakov, S. James, J.-Z. Wu, Discrete fuzzy measures, Springer, 2020.

[3] E. Czogala, J. Drewniak, Associative monotonic operations in fuzzy set theory, Fuzzy sets and systems 12 (3) (1984) pp. 249-269.

[4] Z. Daróczy, Über eine klasse von mittelwerten, Publ. Math. Debrecen 19 (1972) pp. 211-217.

[5] M. Decký, R. Mesiar, A. Stupňanová, Aggregation functions based on deviations, in: International Conference on Information Processing and Management of Uncertainty in KnowledgeBased Systems, Springer, 2018, pp. 151-159.

[6] M. Decký, R. Mesiar, A. Stupňanová, Deviationbased aggregation functions, Fuzzy Sets and Systems 332 (2018) pp. 29-36.

[7] J. Fodor, R. Yager, A. Rybalov, Structure of uninorms, International Journal of Uncertainty, Fuzziness and Knowledge-Based Systems 5 (04) (1997) pp. 411-427.

[8] M. Grabisch, B. De Baets, J. Fodor, On symmetric pseudo-additions and pseudo-multiplications: is it possible to build rings on $[-1,+1]$ ?, in: Proceedings Nineth Internat. Conference on Information Processing and Management of Uncertainty in Knowledge-Based Systems, Annecy, France, Vol. II,, 2002, pp. 1349-1355.

[9] W. Homenda, W. Pedrycz, Symmetrization of fuzzy operators: notes on data aggregation, in: Computational Intelligence for Modelling and Prediction, Springer, 2005, pp. 1-17.

[10] J. Špirková, P. Král', Mixture functions based on deviation and dissimilarity functions, in: International Summer School on Aggregation Operators, Springer, 2019, pp. 255-266.
[11] A. Stupňanová, P. Smrek, Generalized deviation functions and construction of aggregation functions, in: 2019 Conference of the International Fuzzy Systems Association and the European Society for Fuzzy Logic and Technology (EUSFLAT 2019), Atlantis Press, 2019, pp. 96100. 